Pathophysiology of Haemostasis and Thrombosis

\title{
Laboratory diagnosis of thrombophilic states: where do we stand?
}

\author{
Armando Tripodi \\ From the Angelo Bianchi Bonomi Hemophilia and Thrombosis Center, Department of Internal Medicine, University and IRCCS Maggiore Hospital, \\ University of Milano, Italy.
}

\section{Key Words}

Thrombophilia; laboratory investigation; thrombosis

\section{Summary}

Until recently the laboratory diagnosis of thrombophilia consisted on investigation of the plasmatic anticoagulant pathways and the search for dysfibrinogenemia and antiphospholipid antibodies/lupus anticoagulants. More recently, the laboratory investigation has been expanded by including activated protein $C$ (APC) resistance, due or not to the presence of the factor $\mathrm{V}$ Leiden mutation; hyperprothrombinemia, due to the presence of the prothrombin mutation G20210A and hyperhomocysteinemia, due to impairment of the relevant metabolic pathway because of enzymatic and/or vitamin deficiency. Testing for thrombophilia may be useful for many reasons. First, the results of testing may provide valuable information to assess the risk of recurrence in the proband. Second, testing family members is useful for prophylactic and diagnostic purposes. Third, the identification of patients bearing combined defect helps to identify those at increased risk for thrombosis. Testing is recommended for patients with a past history of thrombosis and should be extended to their firstdegree family members. Since most of the tests are not reliable during anticoagulation, it is preferable to postpone laboratory testing until after discontinuation of the treatment. Whenever possible testing should be performed by means of functional assays. DNA analysis is required for the prothrombin mutation G20210A. Laboratory diagnosis for antiphospholipid antibodies/lupus anticoagulant should be performed by a combination of tests including phospholipid-dependent clotting assays and solid phase anticardiolipin antibodies. Hyperhomocysteinemia may be assessed by high-pressure liquid chromatography methods, or by fluorescence polarization immunoassays.

Copyright (C) 2002 S. Karger AG. Basel

\section{Introduction}

Thrombophilic states are those conditions associated with an increased risk of venous thromboembolism. They may be congenital or acquired. Among the congenital conditions the most important are those secondary to deficiencies of the naturally-occurring anticoagulant systems (antithrombin, protein $\mathrm{C}$ or protein $\mathrm{S}$ ) and the presence of mutations affecting coagulation factors such as factor $\mathrm{V}$ or prothrombin. The former may trigger thrombosis because of a loss of function, whereas the latter may trigger thrombosis because of a gain of function. Both lead to a procoagulant imbalance, which enhances

\section{KARGER \\ Fax +4161306 1234 \\ E-Mail karger@karger.ch}

www.karger.com (c) 2002 S. Karger AG. Basel

$1424-8832 / 02 / 0326-0245 \$ 18.50 / 0$
Armando Tripodi

Via Pace 9 - 20122 Milano

Italy

Phone: +3902 55035437

FAX: +390250320723

E-Mail: armando.tripodi@unimi.it 
thrombin generation and ultimately fibrin deposition within the vascular system.

Among the acquired conditions the most important are those secondary to the presence of elevated levels of coagulation factors such as VIII, IX, XI, moderate hyperhomocysteinemia, or the presence of antiphospholipid antibodies. The former may trigger thrombosis because of a gain of function, whereas the mechanisms, which are behind hyperhomocysteinemia and the presence of antiphospholipid antibodies, are still poorly defined. Both the congenital and acquired conditions may be investigated by laboratory methods. This may open new perspectives for the management of patients with thrombosis and may help prevent the occurrence of thrombosis in those who are still asymptomatic.

This review will focus on the most important aspects of the laboratory investigation with special interest on the conditions to be investigated, selection of the subjects, timing of investigation and type of tests to be used.

\section{Conditions to be investigated}

Although many different conditions have been surmised to be associated with an increased risk of venous thromboembolism, a close scrutiny of the literature reveals that some of them such as the defects of the fibrinolytic system and the defect of the naturally-occurring anticoagulant heparin cofactor II have not unequivocally been associated with thrombosis. Furthermore, other candidate conditions such as the defect of the tissue factor pathway inhibitor (TFPI) still remain to be elucidated. Moderate hyperhomocysteinemia due to impairment of the relevant metabolic pathway because of enzymatic and/or vitamin deficiency has also been associated with venous and/or arterial thrombosis and is now included in the thrombophilia work up. Elevated levels of plasmatic coagulation factors, or fibrinolytic inhibitors have been the focus of much attention over the last years as possible causes of increased risk of venous thromboembolism. However, only factor VIII [1] has so far been clearly documented, whereas factor IX [2], XI [3] and the thrombin activatable fibrinolytic inhibitor (TAFI) [4] still remain to be confirmed as risk factors. Therefore, the laboratory should restrict the investigation to antithrombin, protein $\mathrm{C}$ and protein $\mathrm{S}$ defects, to activated protein $\mathrm{C}$ (APC) resistance due or not to the presence of factor $\mathrm{V}$ Leiden, the search for the prothrombin G20210A mutation, the measurement of factor VIII and homocysteine and the search for antiphospholipid antibodies. Special attention should also be paid to the rare cases of dysfibrinogenemia associated with venous and/or arterial thrombosis. Because of the combined effect that multiple conditions may have on the risk of thrombosis, the laboratory investigation should be comprehensive and include all the above conditions.

\section{Subjects to be investigated}

The value of the laboratory investigation for thrombophilia is currently debated. Those who are against argue that the investigation should not be carried out as this does not affect the management of patients with thrombosis [5], whereas those who are in favor argue that the investigation may be of benefit in preventing recurrences in patients with previous events, or in preventing the development of events in asymptomatic individuals such as the relatives of the probands who are discovered incidentally during the family investigation [6]. Both the "no testing at all" and the "testing for all" attitudes should be avoided as the first would deny some benefits to the patients, whereas the second would increase exaggeratedly the cost that should be supported by the health system. Alternatively one should consider selecting carefully the subjects to be investigated in order to maximize the benefit.

The evidence available does not support mass screening of the general population even for those conditions, which are relatively frequent such as APC resistance and the prothrombin mutation. This is valid also for those individuals at increased risk such as women on oral contraceptives, pregnancy, etc. [7]. This attitude is mainly based on the analysis of the cost/benefit ratio [7], but it may change progressively if newer and cheaper global screening procedures become available.

The laboratory investigation should be offered to those patients who experienced previous thrombotic events, especially at young age and without circumstantial risk factors. However, neither age nor the presence/absence of circumstantial risk factors should be taken as the only criterion for the choice of testing, as many thrombophilic patients may develop thrombosis later in their life and/or they may require triggering factors. Because of the beneficial effect (adequate prophylaxis during high-risk situations) which follows the identification of affected subjects who are still asymptomatic, the laboratory investigation should be extended to the first-degree family members of the proband.

\section{Timing of investigation}

Because many of the parameters under investigation are likely to be affected by the acute thrombotic event and/or by the administration of antithrombotic drugs, the laboratory investigation should be performed far from the acute event (at least six months) and few weeks after the discontinuation of oral anticoagulant therapy.

\section{Type of testing}

Whenever possible, testing should be performed with functional assays in order to detect both type I and type II (dys- 
functional) defects. Antigen assays should be carried out to further characterize the defects.

Antithrombin deficiency. This condition should be investigated with heparin cofactor activity assays with thrombin or activated factor $\mathrm{X}$ as target enzymes. The latter enzyme is preferable, as it ensures a better discrimination of carriers from non-carriers of the deficiency and renders the assay insensitive to the presence of heparin cofactor II [8].

Protein $\boldsymbol{C}$ deficiency. The assays which measure the inhibitory activity exerted by APC after activation with snake venom against the natural substrates factor Va and VIIIa in an APTT-based method would be the tests of choice to investigate this condition. However, they are not very specific. Lower than expected protein $\mathrm{C}$ levels have been reported in patients with APC resistance, high factor VIII levels and lupus anticoagulants. A suitable alternative would be the chromogenic assay, which measures the activity exerted by APC after activation with snake venom on small synthetic substrates. This assay is very specific and less prone to artifact. It is suitable to detect most of the protein $\mathrm{C}$ deficiencies, except those rare variants where the defect is restricted to the active site.

Protein $\mathbf{S}$ deficiency. The commercially available functional assays measure the protein $\mathrm{C}$ cofactor activity exerted by protein S in APTT- or PT-based assays. The specificity of these methods is limited. Lower than expected activity has been reported in patients with APC resistance and possibly in patients with high levels of coagulation factors. Until newer and more specific methods become available, the investigation for this condition should be carried out with the antigen measurement. The free antigen should be preferred to the total antigen, as it discriminates better carrier from non-carriers of protein $\mathrm{S}$ congenital deficiency [9].

$A P C$ resistance. There are at least two types of APC resistance. The first is mainly due to the presence of the factor $\mathrm{V}$ Leiden mutation, which renders the mutated factor $\mathrm{V}$ more resistant to the inactivation mediated by APC. The occurrence of other mutations on the factor V gene (i.e., Cambridge) leading to APC resistance (though rare) should be considered. The second (also called phenotypic, or acquired APC resistance) has been documented in many patients without the factor $\mathrm{V}$ Leiden mutation. Though the mechanism is still unclear, acquired APC resistance has been found in association with increased levels of factor VIII, elevated levels of prothrombin, oral contraceptive intake, pregnancy and the presence of lupus anticoagulants. Recent studies have shown that acquired APC resistance is a risk factor for venous thromboembolism and that this risk is not dependent on factor V Leiden [10]. This suggests that the laboratory should undertake both the search for factor V Leiden with DNA analysis and the measurement of APC resistance. The latter can be measured with the method based on paired APTTs performed on undiluted test plasma with and without APC. The test can also be run on test plasma diluted into factor $\mathrm{V}$ deficient plasma. This modification ren- ders the test $100 \%$ specific for factor $\mathrm{V}$ Leiden and can also be run in patients on oral anticoagulants. A suitable flow chart for investigation should involve both the original and the modified test to detect acquired as well congenital (factor V Leiden) APC resistance. Positive (and borderline) cases should then be investigated with DNA analysis to detect the factor V Leiden mutation.

Prothrombin G20210A mutation. This has been identified in the untranslated region of the prothrombin gene and is frequently associated with elevated levels of prothrombin in plasma. Both the presence of the mutation and hyperprothrombinemia are risk factors for venous thromboembolism. Because the measurement of prothrombin alone does not discriminate carriers from non-carriers of the mutation, searching for the mutation with DNA analysis is mandatory.

Factor VIII. This can be measured with clotting as well as with chromogenic methods. The antigen measurement if readily available is also suitable. The choice is mainly cost-related.

Homocysteine. Until recently the test of choice was based on high-pressure liquid chromatography (HPLC). However, newer and simpler immunoassay methods are now available. They are as reliable as the HPLC method [11] and can be used in the clinical laboratory to investigate thrombophilic patients. Fasting homocysteine may be sufficient to screen patients however, the methionine loading test may increase the diagnostic efficacy. The search for the MTHFR mutation which is frequently (though not invariably) associated with hyperhomocysteinemia is not strictly required to investigate thrombophilic patients.

Search for antiphospholipid antibodies. Because of the lack of specific tests, the search must be carried out with two procedures. The first is aimed at identifying solid-phase antiphospholipid antibodies (anticardiolipin, antiBeta2-glycoprotein I, or both); the second is aimed at identifying lupus anticoagulants (LA). This requires a three-step procedure [12]. The screening by means of phospholipid-dependent coagulation tests such as APTT or similar tests and/or diluted Russell viper venom tests (dRVVT); in the presence of LA one or both of the above should be prolonged. The mixing study where the abnormal test will be repeated on a mixture of patient and normal plasma; in the presence of LA the test should be prolonged. The confirmation where the abnormal test will be repeated on the test plasma after supplementation of phospholipids; in the presence of LA the test should be (nearly) normalized. 


\section{References}

1. Koster T, Blann AD, Briet E, Vandenbroucke JP, Rosendaal FR. Role of clotting factor VIII in effect of von Willebrand factor on occurrence of deep-vein thrombosis. The Lancet 1995; 345: 152-55.

2. Van Hylckama Vlieg A, van der Linden IK, Bertina RM, Rosendaal FR. High levels of factor FIX increase the risk of venous thrombosis. Blood 2000; 95: 3678-82.

3. Meijers JCM, Tekelenburg WLH, Bouma BN, Bertina RM, Rosendaal FR. High levels of factor FXI as a risk factor for venous thrombosis. N Engl J Med 2000; 342: 696-701.

4. Van Tilburg NH, Rosendaal FR, Bertina RM. Thrombin activatable fibrinolysis inhibitor and the risk of deep vein thrombosis. Blood 2000; 95: 2855-9.

5. Greaves M, Baglin T. Laboratory testing for heritable thrombophilia: impact on clinical management of thrombotic disease. Brit $\mathrm{J}$
Haematol 2000; 109: 699-703.

6. Mannucci PM. Genetic hypercoagulability: prevention suggests testing family members. Blood 2001; 98: 21-22.

7. Vandenbroucke JP, van der Meer FJM, Helmerhorst FM, Rosendaal FR. Factor V Leiden: should we screen oral contraceptive users and pregnant women? Brit Med J 1996; 313: $1127-30$

8. Demers C, Henderson P, Blajchman MA, Wells MJ, Mitchell L, Johnston M, et al. An antithrombin III assay based on factor Xa inhibition provides a more reliable test to identify congenital antithrombin III deficiency than an assay based on thrombin inhibition. Thromb Haemost 1993; 69: 231-5.

9. Simmonds RE, Ireland H, Lane DA, Zoller B, Garcia de Frutos P, Dahlback B. Clarification of the risk for venous thrombosis associated with hereditary protein $\mathrm{S}$ deficiency by inves- tigation of a large kindred with a characterized gene defect. Ann Intern Med 1998; 128: 8-14.

10. de Visser MCH, Rosendaal FR, Bertina RM. A reduced sensitivity for activated protein $\mathrm{C}$ in the absence of factor $\mathrm{V}$ Leiden increases the risk of venous thrombosis Blood 1999; 93: 1271-6.

11. Tripodi A, Chantarangkul V, Lombardi R, Lecchi A, Mannucci PM, Cattaneo M. Multicenter study of homocysteine measurement. Performance characteristics of different methods, influence of standards on interlaboratory agreement of results. Thromb Haemost 2001; 85: 291-5.

12. Brandt JT, Triplett DA, Alving B, Scharrer IM. Criteria for the diagnosis of lupus anticoagulants: an update [Review]. Thromb Haemost $1995 ; 74 ; 1185-90$. 